\title{
Antigènes de rejet des tumeurs et immunothérapie spécifique du cancer
}

\section{Thierry Boon \\ Vincent G. Brichard Benoît Van den Eynde}

\author{
ADRESSE \\ T. Boon: professeur. V.G. Brichard: chargé de \\ recherches du Fonds national de la recherche \\ scientifique. B. Van den Eynde: docteur en \\ médecine et en sciences. Institut Ludwig pour la \\ Recherche sur le cancer, 74 , avenue Hippo- \\ crate -UCL 74.59, B-1200 Bruxelles, Bel- \\ gique et Unité de génétique cellulaire, \\ International institute of cellular and mole- \\ cular pathology (ICP) et université catho- \\ lique de Louvain, B-1200 Bruxelles, Bel- \\ gique.
}

Des antigènes reconnus sur des tumeurs par des lymphocytes $T$ cytolytiques peuvent servir de cibles à des réponses immunitaires qui détruisent la tumeur sans affecter les tissus normaux. Au cours des dernières années, on a identifié plusieurs gènes qui codent pour ces antigènes. Le premier, $M A G E-1$, codait pour un antigène reconnu sur un mélanome par un clone $T$ cytolytique autologue. Il appartient à une famille d'au moins douze membres, mais la fonction des protéines correspondantes est encore inconnue. Ces gènes ne sont pas exprimés dans les tissus normaux, à l'exception du testicule et, pour certains, du placenta. Des peptides dérivés des protéines codées par ces gènes sont présentés aux lymphocytes $T$ cytolytiques par des molécules HLA spécifiques. Les lymphocytes $T$ cytolytiques de certains malades atteints de mélanome reconnaissent, en outre, des antigènes de différenciation présents sur les tissus normaux. La connaissance précise des antigènes tumoraux permet d'envisager une immunothérapie anticancéreuse spécifique et d'en tester l'efficacité.

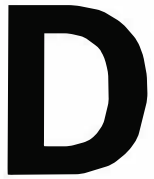
e nombreux travaux effec tués sur des tumeurs de souris, induites au moyen de carcinogènes chimiques ou de rayonnement ultraviolet, ont montré que la plupart de ces tumeurs portent des antigènes capables de provoquer une réponse immunitaire de rejet $[1,2]$. D'autres tumeurs, telles que les tumeurs spontanées, en paraissent au contraire complètement dépourvues [3]. Cependant, nous avons observé que des cellules tumorales, non immunogènes, c'est-à-dire complètement incapables d'induire une réponse immunitaire, peuvent être traitées in vitro par un mutagène pour obtenir des cellules tumorales variantes porteuses d'antigènes très puissants, que nous avons appelées tum- [4]. Ces antigènes déclenchent chez les souris à qui l'on injecte ces cellules variantes une réponse immunitaire qui élimine les cellules tumorales. En outre, les souris qui ont rejeté ces cellules variantes sont devenues capables de rejeter également la cellule tumorale initiale. Nous avons conclu que ces cellules tumorales 


\section{RÉFÉRENCES}

1. Prehn RT, Main JM. Immunity to methylcholanthrene-induced sarcomas. I Natl Cancer Inst 1957; 18 : 769-78.

2. Kripke ML. Immunologic mechanisms in UV radiation carcinogenesis. Adv Cancer Res $1981 ; 34: 69-106$.

3. Hewitt H, Blake E, Walder A. A critic of the evidence for active host defense against cancer based on personal studies of 27 murine tumors of spontaneous origin. $\mathrm{Br} J$ Cancer $1976 ; 33: 241-59$.

4. Van Pel A, Vessière F, Boon T. Protection against two spontaneous mouse leukemias conferred by immunogenic variants obtained by mutagenesis. I Exp Med 1983; 157: 1992-2001.

5. Boon T, Van Snick J, Van Pel A, Uyttenhove $C$, Marchand $M$. Immunogenic variants obtained by mutagenesis of mouse mastocytoma P815. II. T lymphocyte-mediated cytolysis. J Exp Med 1980; 152: 118493.

6. Townsend A, Bastin J, Brownlee G. Cytotoxic $T$ lymphocytes recognize influenza hemagglutinin that lacks a signal sequence. Nature $1986 ; 324: 575-7$.

7. Lurquin C, Van Pel A, Mariamé B, De Plaen E, Szikora JP, Janssens C, Reddehase $\mathrm{M}$, Lejeune J, Boon T. Structure of the gene coding for tum- transplantation antigen P91A. A peptide encoded by the mutated exon is recognized with Ld by cytolytic $\mathrm{T}$ cells. Cell 1989; $58: 293-303$.

8. Mandelboim O, Berke G, Fridkin M, Feldman $M$, Eisenstein $M$, Eisenbach $L$. CTL induction by a tumour-associated antigen octapeptide derived from a murine lung carcinoma. Nalure 1994; 369: 67-71

9. Van den Eynde B, Lethé B, Van Pel A, De Plaen $E$, Boon $T$. The gene coding for a major tumor rejection antigen of tumor P815 is identical to the normal gene of syngeneic DBA/2 mice. J Exp Med 1991; 173 : 1373-84.

10. Mukherji B, MacAlister TJ. Clonal analysis of cytotoxic $T$ cell response against human melanoma. $J$ Exp Med 1983; 158: non immunogènes portent un antigène trop faible pour induire une réponse immunitaire mais qui peut servir de cible à une réponse immunitaire préalablement induite. Cette réponse implique des lymphocytes $\mathrm{T}$. Une explication possible de cette protection contre la tumeur parentale par les variants tum-serait que les lymphocytes $\mathrm{T}$ dirigés contre l'antigène tumoral sont de trop faible affinité pour être activés par cet antigène mais qu'ils voient leur activation facilitée par des lymphokines sécrétées par les lymphocytes $\mathrm{T}$ de forte affinité dirigés contre le nouvel antigène présent sur le variant tum-. Nous avons obtenu le même résultat avec toutes les tumeurs de souris que nous avons testées. Nous sommes donc arrivés à la conclusion que toutes les tumeurs de souris portent des antigènes de rejet tumoral. Il nous a dès lors paru raisonnable d'espérer que les cellules cancéreuses humaines

portent également ce type d'antigène et que l'on puisse déclencher artificiellement chez les patients cancéreux une réponse de lymphocytes $\mathrm{T}$ dirigés contre ces antigènes.

\section{Nature des antigènes de rejet des tumeurs}

L'analyse des antigènes de rejet des tumeurs de souris a pu être poursuivie in vitro grâce à l'obtention de lymphocytes $\mathrm{T}$ cytolytiques capables de détruire les cellules qui portent les antigènes qu'ils recomnaissent [5]. La nature des antigènes reconnus par ces lymphocytes $\mathrm{T}$ cytolytiques antitumoraux a pu être établie par une approche génétique. L'ADN des cellules exprimant ces antigènes a été transfecté dans d'autres cellules et des transfectants exprimant les antigènes ont été identifiés par leur capacité de stimuler les lymphocytes $\mathrm{T}$ dirigés contre ces antigènes. Ainsi,

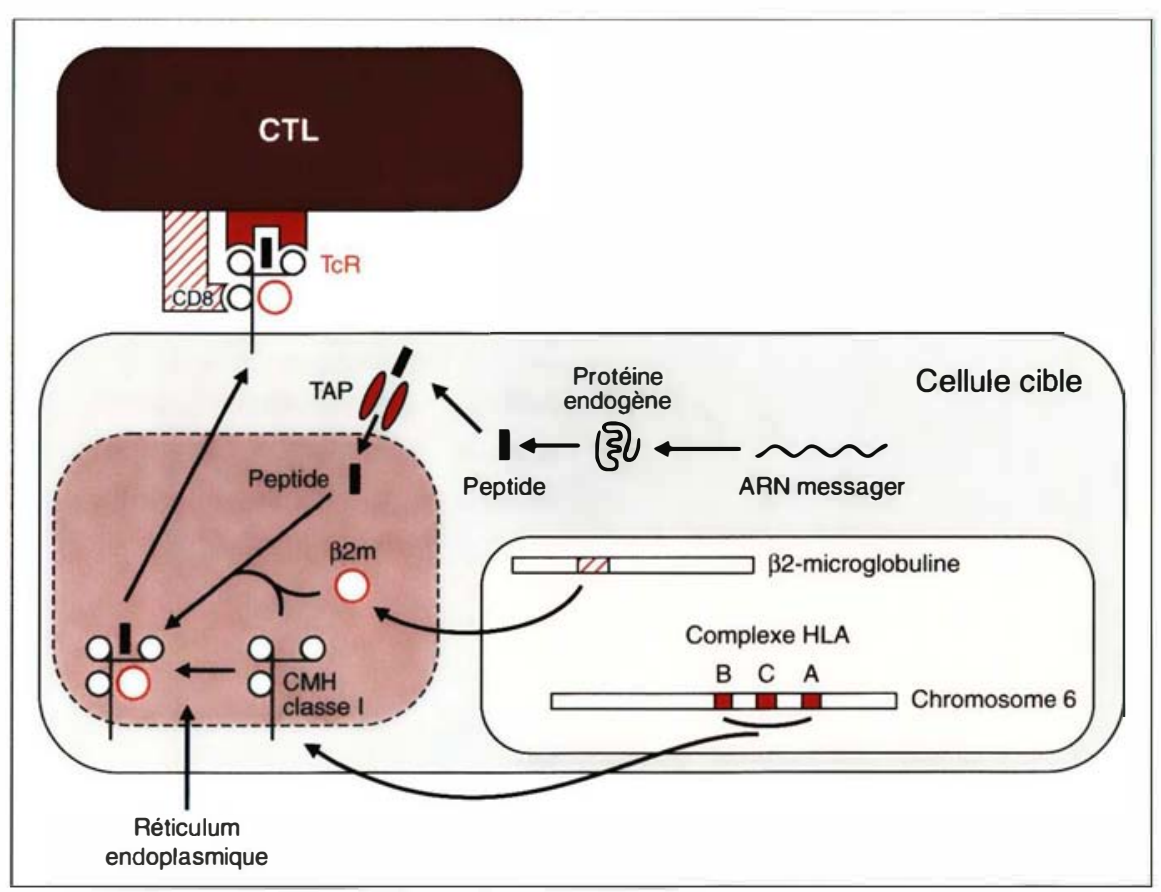

Figure 1. Présentation du peptide antigénique par les molécules du CMH-I aux lymphocytes $\mathrm{CDB}^{+}$cytolytiques. Une protéine endogène est dégradée en petits fragments (peptides) apportés dans le réticulum par un transporteur de peptide (TAP) pour y être associés à des molécules de classe I du CMH et à la $\beta 2$-microglobuline. Ce complexe migre à la surface de la cellule pour être reconnu par un Iymphocyte $T$. Le récepteur du CTL (TcR, en rouge) reconnaît à la fois le peptide et la molécule CMH: pour qu'un antigène soit reconnu sur une cellule, il faut que le gène correspondant soit exprimé afin de produire le bon peptide, mais aussi que la molécule CMH appropriée soit présente. 


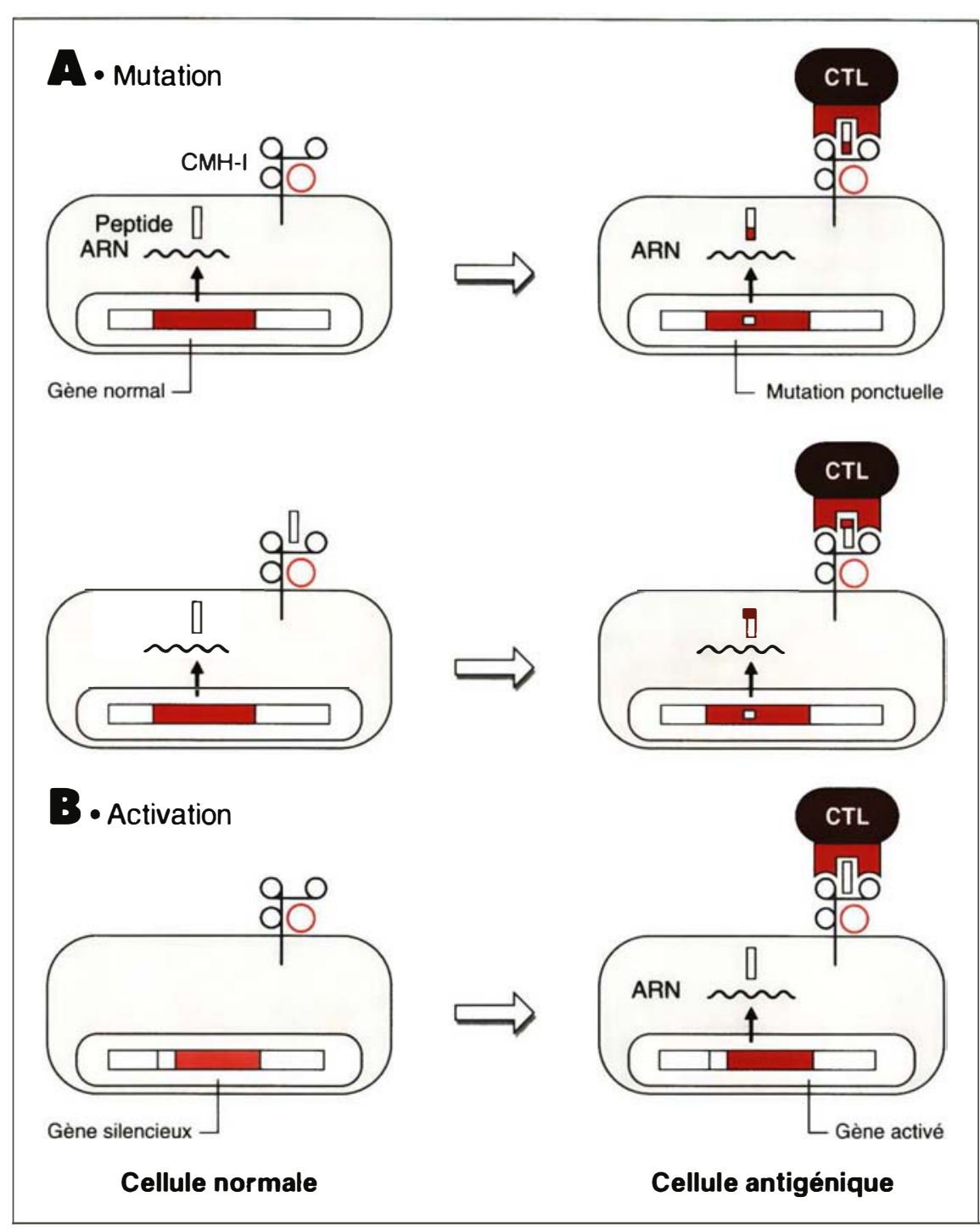

Figure 2. Mécanismes d'apparition d'antigènes tumoraux. A. Mutation. La protéine codée par un gène exprimé dans les cellules normales ne donne pas de peptide présentable par le CMH-I. Si une mutation dans le gène entraîne un changement d'acide aminé dans le peptide issu de la protéine, celui-ci devient alors présentable par le $\mathrm{CMH}-\mathrm{l}$ et forme un antigène reconnu par un lymphocyte. Certains gènes exprimés dans des cellules normales produisent un peptide capable de se lier à une molécule $\mathrm{CMH}$-I mais la tolérance naturelle élimine les lymphocytes $T$ cytolytiques (CTL) qui les reconnaissent. En revanche, si une mutation affecte le peptide, celui-ci se lie encore au CMH-I mais forme alors un antigène différent, reconnu par d'autres CTL. B. Activation. Le gène codant pour l'antigène ne s'exprime pas dans les cellules normales. Dans la tumeur, le gène s'exprime et un peptide issu de la protéine ainsi transcrite peut se lier à une molécule $\mathrm{CMH}-$ l et l'ensemble être reconnu par les CTL.

$\mathrm{m} / \mathrm{s} n^{\circ}$ 9, vol. 11 , septembre 95 nous avons pu isoler les gènes codant ces antigènes. Pendant que nous arrivions à ce résultat, A. Townsend démontrait que les protéines virales peuvent être reconnues par les lymphocytes $T$ même lorsqu'elles n'apparaissent pas à la surface de la cellule [6]. En effet, ces protéines peuvent être fragmentées en petits peptides, qui sont alors transportés dans le réticulum endoplasmique où ils vont se lier à des molécules de classe I codées par le complexe majeur d'histocompatibilité (C.MHI). Les complexes ainsi formés sont transportés à la surface de la cellule et ce sont eux qui sont reconnus par le récepteur du lymphocyte $\mathrm{T}$ (figure 1).

Nous avons montré que ce processus n'est pas réservé aux protéines virales, mais qu'il s'applique également aux protéines codées par le génome de la cellule elle-même. Nos cellules disposent donc d'un mécanisme remarquable qui leur permet de faire ausculter à tout moment leur contenu par les lymphocytes $\mathrm{T}$ cytolytiques. ('es lymphocytes peuvent ainsi reconnaître les peptides anormaux et répondre par l'élimination de la cellule. Il peut donc exister une véritable surveillance immunitaire de l'intégrité et de l'expression correcte du génome cellulaire.

Dans quelles conditions peuvent apparaître à la surface des cellules cancéreuses des antigènes qui ne sont pas présents sur les cellules normales? Nous avons identifié deux mécanismes principaux. Le premier est la mutation (figure 2). Un gène exprimé dans les cellules normales ne produit pas d'antigène car la protéine codée par ce gène ne produit pas de peptide pouvant se lier à une molécule d'histocompatibilité de classe I $\left(m / s n^{\circ} 1, v o l .8, p .80\right)[7]$. Une mutation dans le gène change un acide aminé dans la protéine, qui produit dès lors un peptide capable de se lier à une molécule de classe I pour former un antigène reconnu par un lymphocyte. Il existe une variante de ce mécanisme. Certains gènes exprimés dans toutes les cellules produisent un peptide qui se lic à une molécule de classe I. Mais précisément parce que l'antigène est exprimé dans les cellules normales, le processus de tolérance naturelle élimine les lymphocytes $\mathrm{T}$ cytoly- 


\section{RÉFÉRENCES}

11. Hérin M, Lemoine C, Weynants $P$, Vessière F, Van Pel A, Knuth A, Devos R, Boon $T$. Production of stable cytolytic $T$-cell clones directed against autologous human melanoma. Int J Cancer 1987; 39: 390-6.

12. Degiovanni $G$, Hainaut $P$, Lahaye $T$, Weynants $P$, Boon $T$. Antigens recognized on a melanoma cell line by autologous cytolytic $\mathrm{T}$ lymphocytes are also expressed on freshly collected tumor cells. Lur J Immunol $1990 ; 20: 1865-8$.

13. Topalian SL, Solomon D, Davis FP, Chan AE, Freerdsen DI, Linehan WM, Lot ze MT, Robertson CN, Seipp CA, Simon P, Simpson CG, Rosenberg SA. Immunotherapy of patients with advanced cancer using py of patients with advanced cancer using binant interleukin-2: a pilot study. I Clin Oncol 1988; 6 : 839-53.

14. Van den Eynde B, Hainaut P, Hérin $\mathbf{M}$, Knuth A, Lemoine C, Weynants $P$, van der Bruggen P, Fauchet R, Boon T. Presence on a human melanoma of multiple antigens recognized by autologous CTL. Int / Cancer $1989 ; 44: 634-40$

15. Wölfel T, Hauer M, Klehmann E, Brichard V, Ackermann B, Knuth A, Boon T, Meyer zum Büschenfelde KH. Analysis of antigens recognized on human melanoma cells by A2-restricted cytolytic T lymphocytes (CTL.). Int J Cancer 1993; 55 : 237-44.

16. van der Bruggen $P$, Traversari C, Chomez $P$, Lurquin $C$, De Plaen $E$, Van den Eynde B, Knuth A, Boon T. A gene encoding an antigen recognized by cytolytic $T$ lymphocytes on a human melanoma. Science $1991 ; 254: 1643-7$

17. Traversari $G$, van der Bruggen P, Luescher IF, Lurquin C, Chomez P, Van Pel A De Plaen E, Amar-Costesec A, Boon T. A nonapeptide encoded by human gene MAGE-l is recognized on HLA-Al by cytolytic $T$ lymphocytes directed against tumor antigen MZ2-E. J Exp Med 1992; 176: 14537 .

18. De Plaen E, Arden K, Traversari C Gaforio JJ, Szikora JP, De Smet C, Brasseur $F$, van der Bruggen $P$, Lethé $B$, Lurquin $C$, Brasseur R, Chomez P, De Backer O, Cavenee $W$, Boon T. Structure, chromosomal localization and expression of twelve gene of the MACE family. Immunogenetics 1994 ; tiques qui le reconnaissent. Cependant, une mutation affectant le gène peut produire un nouveau peptide qui se lie encore à la molécule de classe I mais forme un antigène différent. Celui-ci peut être reconnu par des lymphocytes $\mathrm{T}$ qui n'ont pas été éliminés par le processus de tolérance (figure 2). Il nous paraît probable que des antigènes de rejet des tumeurs peuvent apparaître à la suite de mutations. En effet, les tumeurs contiennent souvent des oncogènes mutés ou des protéines p53 mutées. Un antigène de tumeur de souris récemment identifié comporte d'ailleurs un acide aminé muté [8].

Un autre mécanisme paraît responsable de l'apparition de nombreux antigènes spécifiques de tumeurs. Ici, le gène qui code l'antigène n'est pas exprimé dans les cellules normales. Dans la tumeur, cependant, ce gène est transcrit et un peptide antigénique est dérivé de sa protéine. Le premier exemple d'um de ces gènes est le gène PlA qui code pour un antigène reconnu par des lymphocytes $T$ cytolytiques sur un mastocytome de la souris [9]. Ce gène semble jouer un rôle dans la transformation tumorale. Il est d'ailleurs exprimé dans plusieurs mastocytomes de la souris. Le gène PlA n'est pas exprimé dans les cellules normales de souris à l'exception de certaines cellules du testicule et du placenta. Il nous paraît probable que, dans ces tissus, l'expression du gène n'entraîne pas l'expression de l'antigène. En effet, des cellules telles que les précurseurs des spermatozoïdes n'expriment pas les molécules d'histocompatibilité et ne peuvent donc pas présenter des antigènes. Il s'avère d'ailleurs que des souris mâles immunisées contre l'antigène codé par $P I A$ restent fertiles et ne montrent aucun signe d'inflammation testiculaire.

\section{Antigènes du mélanome humain}

Pour l'analyse des antigènes reconnus par des lymphocytes $\mathrm{T}$ sur des tumeurs humaines, de nombreux travaux se sont portés sur le mélanome, car cette tumeur se met plus facilement en culture que d'autres. On peut irradier pour les tuer des cellules de mélanome en culture et les mêler à des lymphocytes autologues, c'est-à-dire des lymphocytes obtenus à partir du sang du même malade Après deux à quatre semaines de cette culture mixte tumeur-lymphocytes, les lymphocytes dirigés contre un antigène tumoral peuvent proliférer et se différencier en lymphocytes $\mathrm{T}$ cytolytiques (figure 3). Si l'on dilue les lymphocytes répondeurs tout en les restimulant par des cellules tumorales, on peut obtenir des cultures de lymphocytes issus d'un seul lymphocyte cytolytique. L'obtention de tels clones de lymphocytes $\mathrm{T}$ cytolytiques s'est avérée essentielle pour le progrès des travaux sur les tumeurs de souris et sur les tumeurs humaines, car ces clones de lymphocytes T cytolytiques sont très actifs et très spécifiques (figure 3) $[10,11]$. Dans certains cas, il a été possible de montrer que ces clones CTL reconnaissent aussi des échantillons tumoraux fraîchement prélevés, indiquant ainsi que l'antigène cible n'est pas le résultat d'un artefact de culture [12, 13]. Avec les clones cytolytiques on peut faire des immunosélections. Quelques millions de cellules tumorales sont incubées 24 heures en présence de quelques millions de lymphocytes d'un clone donné. La quasi-totalité des cellules tumorales sont tuées, mais il reste parfois quelques cellules tumorales qui ont résisté aux CTL. En général, elles ont résisté car elles ont perdu l'antigène reconnu par les CTL (figure 3). On peut alors rechercher si ces variants de perte résistent également aux autres clones cytolytiques dirigés contre la même tumeur. Souvent ce n'est pas le cas, ce qui indique que ces clones reconnaissent un autre antigène. Nous sommes arrivés ainsi à la conclusion qu'au moins six antigènes différents étaient reconnus par les (CTL sur un mélanome [14]. Cette observation, que nous avions déjà faite sur des tumeurs de souris, a été étendue à d'autres tumeurs humaines [15].

\section{Les gènes MAGE}

Nous avons pu identifier un premier gène codant pour un antigène reconnu sur un mélanome par un clone $\mathrm{T}$ cytolytique autologue [16]. Pour cela, nous avons isolé l'ADN des cellules et nous l'avons transfecté dans le variant de perte immunosélectionné. Quelques rares transfectants se 


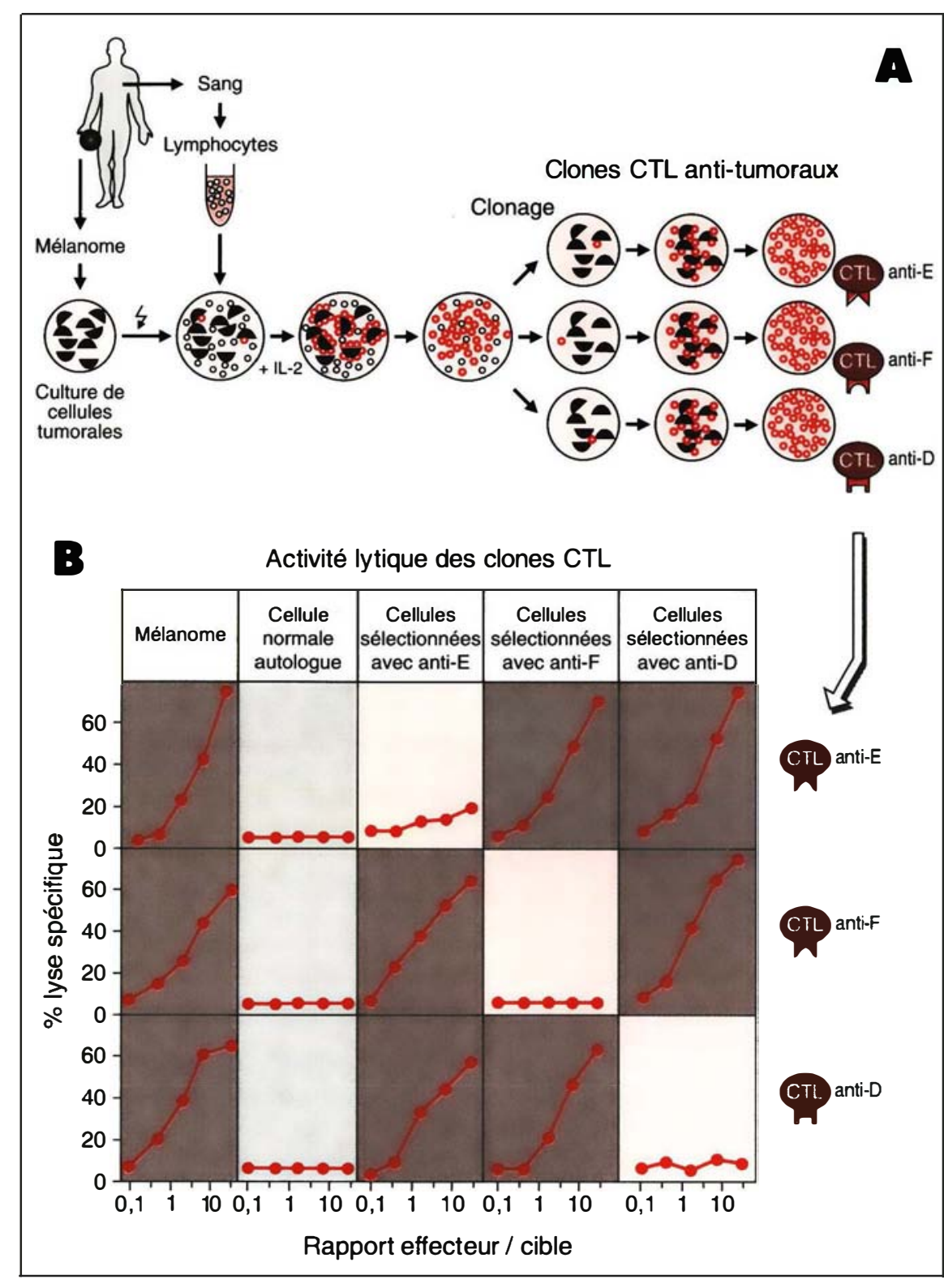

sont avérés capables de stimuler le clone cytolytique. En répétant l'opération avec une bibliothèque de cosmides, nous avons pu obtenir à nouveau ce type de transfectants et isoler le gène que nous avons appelé MAGE-1 ( M M pour mélanome, «AGE» pour antigène).

Le gène $M A G E-1$ contient trois exons et sa partie codante est située entièrement dans le troisième exon. Il ne ressemble à aucun gène connu et sa séquence est identique à celle du même gène présent dans les cellules normales du malade. L'antigène tumoral pour lequel il code n'est donc pas le résultat d'une mutation mais bien de l'activation sélective du gène dans le mélanome. Nous avons pu établir que l'antigène consiste en un nonapeptide présenté par la molécule d'histocompatibilité HLAA 1, molécule portée par le quart de la population européenne (figure 4) [17].

Le gène $M A G E-1$ fait partie d'une famille d'au moins douze gènes très semblables situés sur le chromosome $X$ [18]. Parmi ces douze gènes, six sont exprimés dans un certain
Figure 3. Obtention de clones CTL antitumoraux autologues. A. Clonage par dilution limite: Après 21-28 jours de culture mixte de lymphocytes et de cellules tumorales autologues irradiées (MLTC), une population effectrice spécifique de la tumeur est clonée par dilution limite. La spécificité des clones CTL se traduit par la reconnaissance de la tumeur autologue et une absence de reconnaissance de lymphocytes $B$ ou de fibroblastes autologues. B. Un variant de perte $d^{\prime}$ antigène est sélectionné par co-culture avec un clone CTL et défini par son absence de reconnaissance par ce clone CTL. La présence $d$ 'autres antigènes sur ce variant se traduit par sa sensibilité à la lyse par d'autres clones de CTL. nombre de tumeurs. Les six gènes ont une structure très semblable (70\% à $99 \%$ d'identité) en ce qui concerne la partie qui code pour la protéine ( 300 acides aminés). En revanche, au début des gènes, les différences sont beaucoup plus accentuées, particulièrement au niveau du premier exon et de son promoteur. Le gène $M A G E-4$ contient huit exemplaires du premier exon précédés de promoteurs différents et fonctionnant par épissage alternatif. Il semble que la duplication du gène $M A G E$ en une nombreuse famille a pour résul- 


\section{RÉFÉRENCES}

19. Brasseur F, Marchand M, Vanwijck R, Hérin $\mathrm{M}$, Lethé $\mathrm{B}$, Chomez $\mathrm{P}$, Boon $\mathrm{T}$. Human gene MAGE-1, which codes for a tumor rejection antigen, is expressed by some breast tumors. Int J Cancer 1992; 52: 839-41.

20. Weynants P, Lethé B, Brasseur F, Marchand $\mathrm{M}$, Boon $\mathrm{T}$. Expression of $M A G E$ genes by non-small-cell lung carcinomas. Int f Cancer $1994 ; 56: 826-9$.

21. Patard J, Brasseur F, Gil-Diez S, Radvanyi F, Marchand M, François P, Abi Aad A, Van Cangh P, Abbou CC, Chopin D, Boon $\mathrm{T}$. Expression of MAGE genes in transitional-cell carcinomas of the urinary bladder. Int $\int$ Cancer 1995 , sous presse.

22. Gaugler B, Van den Eynde B, van der Bruggen P, Romero P, Gaforio IJ, De Plaen $E$, Lethé B, Brasseur F, Boon T. Human gene $M A G E-3$ codes for an antigen recognized on a melanoma by autologous cytolytic T lymphocytes. $\int$ Exp Med 1994; 179 : 921-30.

23. van der Bruggen $P$, Bastin J, Gajewski T Coulie PG, BoëPß, De Smet C, Traversari C Townsend A, Boon T. A peptide encoded by human gene $M A G E-3$ and presented by HLA-A2 induces cytolytic T lymphocytes that recognize tumor cells expressing MAGE-3. Eur J Immunol 1994; 24: 3038-43.

24. Brichard V, Van Pel A, Wölfel T, Wölfel $\mathrm{C}$, De Plaen E, Lethé B, Coulie P, Boon T. The tyrosinase gene codes for an antigen recognized by autologous cytolytic $T$ lymphocytes on HLA-A2 melanomas. J Exp Med 1993 ; 178 : 489-95.

25. Topalian SL, Rivoltini L, Mancini M, Markus NR, Robbins PF, Kawakami Y, Rosenberg SA. Human CD $4^{+} \mathrm{T}$ cells specifically recognize a shared melanoma-associated antigen encoded by the tyrosinase gene. Proc Natl Acad Sci USA 1994; 91 : 9461-9465.

26. Coulie PG, Brichard V, Van Pel A, Wölfel $T$, Schneider J, Traversari C, Mattei S De Plaen E, Lurquin C, Szikora JP, Renauld

$\mathrm{JC}$, Boon T. A new gene coding for a differentiation antigen recognized by autologous cytolytic T lymphocytes on HLA-A2 tat de mettre la même fonction sous le contrôle de nombreux promoteurs différents, ce qui permet d'assurer une expression très sélective dans certaines cellules et à certains moments. Un argument en faveur de cette hypothèse est que les zones hydrophobes et hydrophiles des protéines de la famille MAGE sont remarquablement conservées, ce qui suggère que toutes ces protéines ont bien la même fonction. Malheureusement nous ignorons encore tout de cette fonction.

Même lorsqu'on emploie la technique PCR, qui est très sensible, on n'observe aucune expression des gènes $M A G E$ dans les tissus normaux, à l'exception de cellules du testicule et, pour certains gènes MAGE, du placenta. L'expression remarquablement sélective des gènes $M A G E$ dans les tumeurs devrait permettre leur utilisation en immunothérapie anticancéreuse. Le gène $M A G E-1$ est exprimé dans à peu près $35 \%$ des mélanomes. Il est également exprimé dans des tumeurs de la sphère ORL, du sein, de la vessie et du poumon (Tableau I) [19-21].

Outre ce premier antigène constitué d'un peptide MAGE-l présenté par HLA-Al, nous avons observé que MAGE-1 produit un autre peptide qui se lie à une molécule d'histocompatibilité HLA-C pour former un second antigène. Le gène $M A G E-3$ produit également un peptide reconnu par des CTL sur HLA-Al et un autre reconnu sur HLA-A2 [22, 23] (figu$r e 4)$. Nous avons également identifié deux autres gènes, que nous avons nommés BAGE et $G A G E$ parce qu'ils ne s'expriment pas non plus dans les tissus normaux à l'exception des testicules. Chacun de ces deux gènes code également pour un antigène reconnu par des lymphocytes $\mathrm{T}$ cytolytiques.

\section{Antigènes de différenciation}

Outre ces antigènes très spécifiques des tumeurs, nous avons observé que les lymphocytes $T$ cytolytiques de certains malades atteints de mélanome reconnaissent des antigènes de différenciation portés non seulement par les mélanomes mais également par les mélanocytes normaux. Le gène codant pour la tyrosinase, une enzyme qui intervient dans la synthèse de la mélanine, produit au moins trois peptides antigéniques différents, dont deux sont présentés par la molécule HLA-A2, portée par la moitié de la population européenne [24]. Le gène codant pour la tyrosinase produit également un peptide présenté par HLA-DR4 et reconnu par des lymphocytes auxiliaires $\mathrm{CD}^{+}$. Cette observation ouvre la perspective d'immuniser contre des épitopes présentés par des molécules HLA de classe I et de classe II, et d'utiliser le rôle critique des lymphocytes auxiliaires dans le déclenchement de la réponse immunitaire [25]

Un autre gène qui, comme le gène codant pour la tyrosinase, ne s'exprime que dans les mélanocytes normaux et dans la plupart des mélanomes, produit également un antigène (figure 4) [26, 27]. D'autres ont montré qu'un autre antigène dérive de la protéine gp100, qui est également spécifique des mélanocytes et des mélanomes [28]. Il n'est pas absolument évident que ces antigènes de différenciation pourront être utilisés pour immuniser des cancéreux, car il pourrait y avoir des effets secondaires inacceptables au cours de réponses contre les mélanocytes normaux dont certains se trouvent dans la choroïde de l'œil.

\section{Immunothérapie anticancéreuse spécifique}

La connaissance précise de certains gènes codant pour des antigènes de rejet des tumeurs ainsi que des peptides antigéniques reconnus par les lymphocytes $\mathrm{T}$ cytolytiques ouvre de nouvelles perspectives pour les essais d’immunothérapie spécifique. Pour comprendre les avantages par rap-

Figure 4. Antigènes tumoraux reconnus par des CTL autologues. Le gène MAGE-1, codant pour un antigène tumoral, fait partie d'une famille comptant au moins 12 membres. Les peptides produits par ces gènes sont présentés aux CTL par des molécules du CMH-I spécifiques. Par exemple, le gène MAGE-1 produit un peptide présenté par HLA-A1 et un autre peptide présenté par HLA$C w^{*} 16$. 
port à la situation antérieure, prenons l'exemple de l'antigène MAGE1 qui est présenté par la molécule HLA-A1. D'abord, on pourra identifier les malades dont la tumeur porte l'antigène: par typage HLA à partir du sang du malade et par amplification PCR pour déceler l'expression du gène $M A G E-1$ sur un très petit échantillon de tumeur congelé immédiatement après la résection chirurgicale. Ces opérations ne devraient pas prendre plus de quelques jours (figure 5). Ensuite, on pourra tenter d'immuniser les malades avec plusieurs formes de l'antigène. Finalement, on pourra évaluer l'effet de ces immunisations, non seulement en examinant l'évolution des malades, mais aussi en testant leurs lymphocytes pour déterminer si on a déclenché une réponse de lymphocytes $\mathrm{T}$ cytolytiques contre l'antigène. Lorsque l'on trouvera des modes d'immunisation permettant d'obtenir régulièrement des réponses lymphocytaires, on pourra entamer de véritables études cliniques pour voir si cette immunité peut retarder ou arrêter l'évolution des cancers.

La proportion des patients atteints de mélanome que l'on pourrait immuniser dès à présent s'obtient en multipliant la fréquence des tumeurs qui expriment un des gènes $M A G E$,

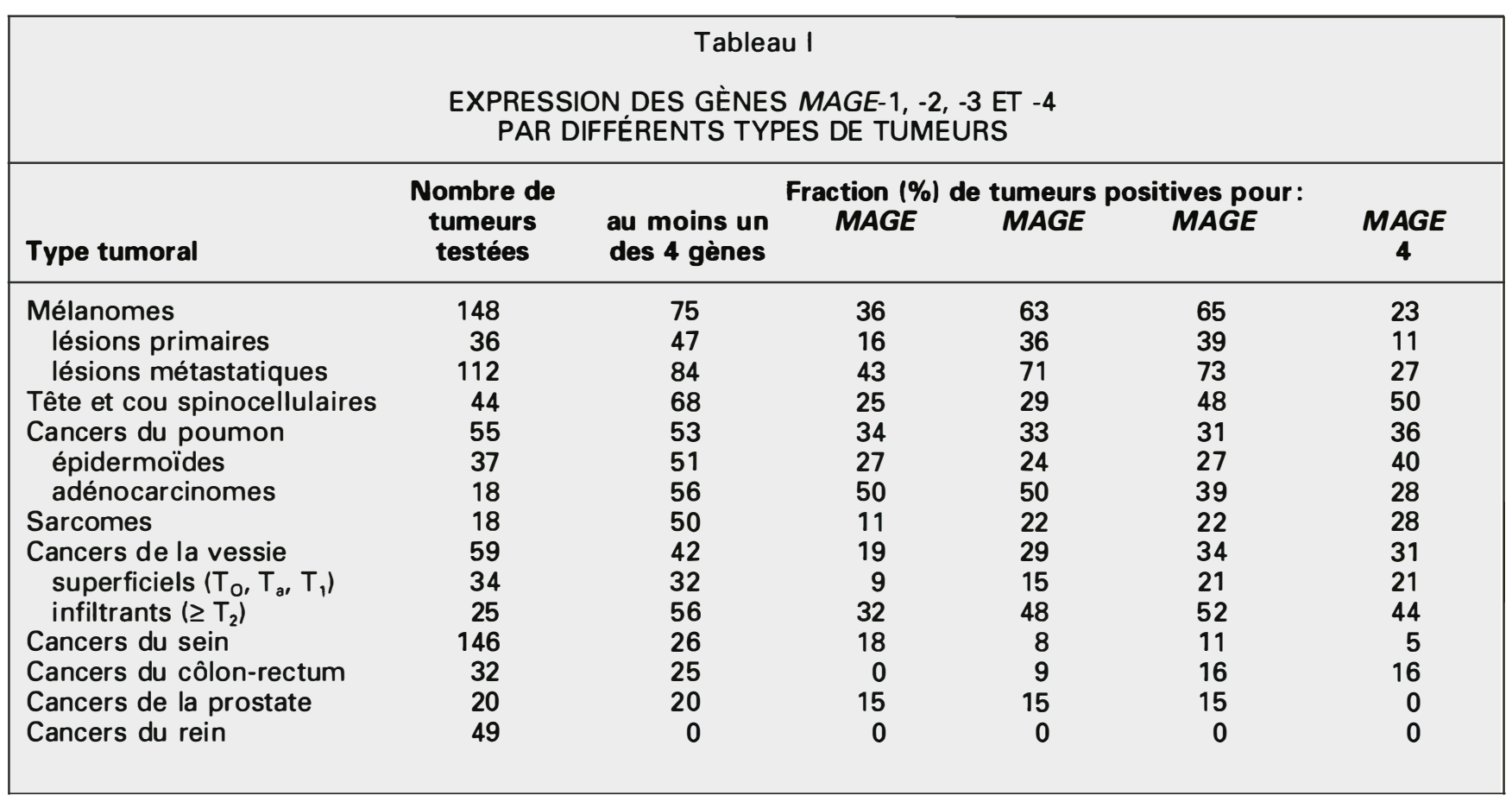

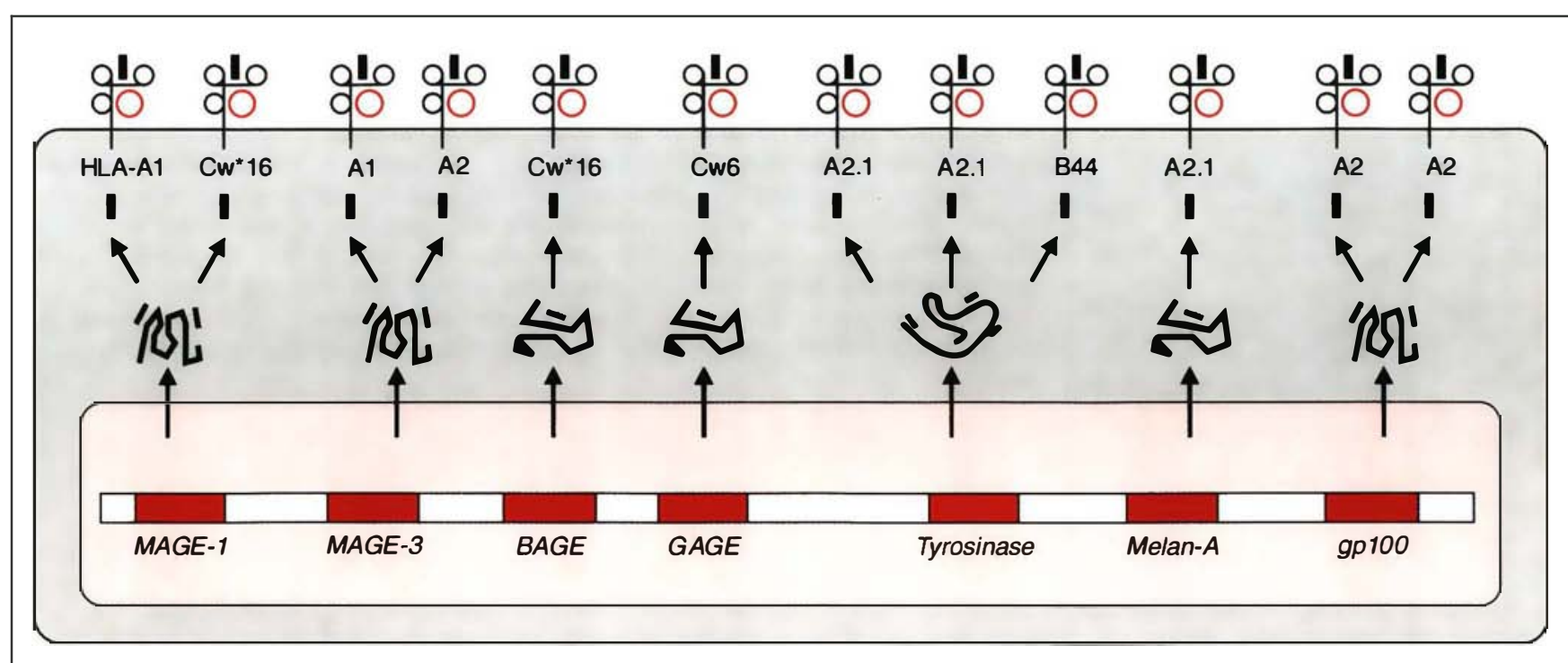




\section{RÉFÉRENCES}

27. Kawakami $\mathrm{Y}$, Eliyahu S, Delgado $\mathrm{CH}$, Robbins PF, Rivoltini L, Topalian SL, Miki $T$, Rosenberg SA. Cloning of the gene coding for a shared human melanoma antigen recognized by autologous $\mathrm{T}$ cells infiltrating into tumor. Proc Natl Acad Sci USA $1994 ; 91$ : 3515-9.

28. Bakker ABH, Schreurs MWJ, de Boer AJ, Kawakami Y, Rosenberg SA, Adema GJ, Figdor CG. Melanocyte lineage-specific antigen gp100 is recognized by melanoma-derived tumor-infiltrating lymphocytes. I Exp Med 1994; 179: 1005-9.

29. Aichele $\mathbf{P}$, Hengartner $\mathrm{H}$, Zinkernagel RM, Schulz M. Antiviral cytotoxic T cell response induced by in vivo priming with a free synthetic peptide. J Exp Med 1990; 171 : 1815-20.

30. Cepko C, Roberts BE, Mulligan RC. Construction and applications of a highly transmissible murine retrovirus shuttle vector. Cell 1984; 37: 1053-62.

31. Rosenfeld MA, Yoshimura K, Trapnell BC, Yoneyama K, Rosenthal ER, Dalemans W, Fukayama M, Bargon J, Stier LE, Stratford-Perricaudet L, Perricaudet M, Guggino WB, Pavirani A, Lecocq JP, Crystal RG. In vivo transfer of the human cystic fibrosis transmembrane conductance regulator gene to the airway epithelium. Cell 1992; 68 : 143-55.

32. Taylor J, Trimarchi C, Weinberg R, Languet $B$, Guillemin $F$, Desmettre $P$, Paoletti E. Efficacy studies on a canarypox-rabies recombinant virus. Vaccine 1991 ; 9: 190-3.

33. Salgaller ML, Weber JS, Koenig S, Yannelli JR, Rosenberg SA. Generation of specific anti-melanoma reactivity by stimulation of human tumor-infiltrating lymphocytes with MAGE-1 synthetic peptide. Cancer Immunol Immunother 1994; 39: 105-16.

34. Lopez-Nevot MA, Esteban F, Ferron A, Gutierrez Oliva MR, Romero C, Huelin C, Ruiz-Cabello F, Gärrido F. HLA class I gene expression on human primary tumours and autologous metastases: demonstration of selective losses of HIA antigens on colorectal, gastric and laryngeal carcinomas. $\mathrm{Br} J$ Cancer $1989 ; 59: 221-6$.

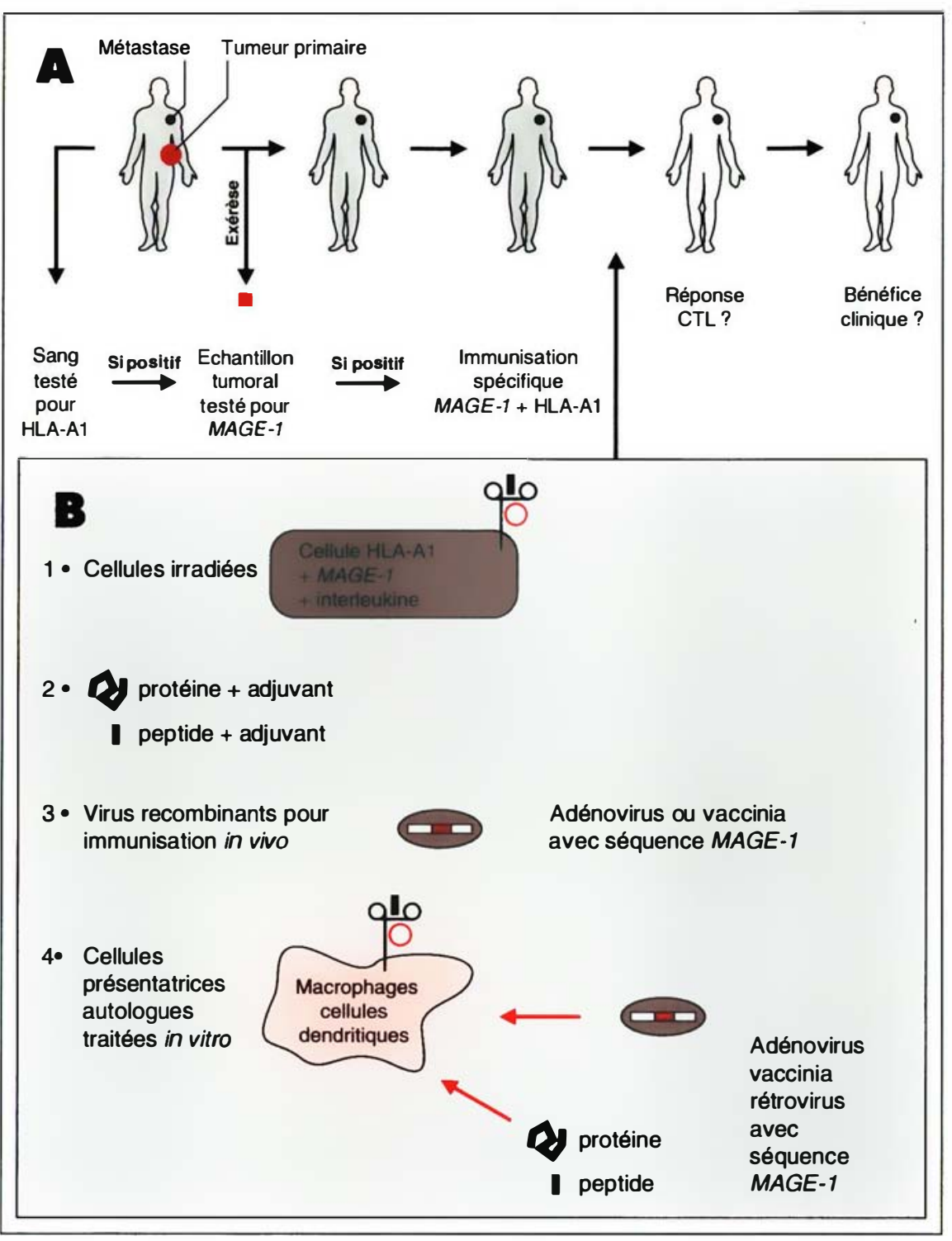

Figure 5. Immunisation: schéma général. A. Typage du malade. Si l'on veut immuniser contre l'antigène dérivé du gène MAGE-1 et présenté par HLA-A1, le malade doit d'abord être typé pour HLA-A1. Sa tumeur est testée pour l'expression du gène MAGE-1. Si ces tests sont positifs, le malade peut bénéficier d'une immunothérapie spécifique pour l'antigène donné. B. Différentes voies d'immunisation sont envisagées: (1) Injection de cellules tumorales exprimant l'antigène, transfectées avec des gènes codant pour des interleukines, puis irradiées. (2) Vaccination par les peptides ou les protéines antigéniques recombinantes associés à un adjuvant. (3) Injection de virus recombinants défectifs porteurs de la séquence codant pour le peptide antigénique. (4) Injection de cellules présentatrices de l'antigène préalablement incubées avec l'antigène ou infectées par un virus recombinant exprimant la séquence MAGE-1. 
$B A G E$ ou $G A G E$ avec la fréquence de personnes qui expriment les types HLA qui présentent leurs antigènes. Dès à présent, à peu près $50 \%$ de patients atteints de mélanome pourraient faire l'objet d'études pilotes d'immunisation.

En ce qui concerne les modes d'immunisation, de nombreuses possibilités seront explorées (figure 5). Une première approche serait l'injection de cellules irradiées exprimant l'antigène. Ces cellules pourraient être transfectées avec des gènes codant pour des interleukines afin de les rendre plus immunogènes. Disposant du gène $M A G E-1$, il serait également possible de produire la protéine en grande quantité en faisant exprimer le gène dans des bactéries ou dans des levures. Cette protéine, combinée à un adjuvant approprié, pourra alors servir de vaccin. Le peptide antigénique lui-même pourra être synthétisé et utilisé comme vaccin [29]. Une autre approche qui paraît intéressante consistera à obtenir du malade des cellules qui présentent très bien les antigènes aux lymphocytes T, c'est-à-dire des macrophages ou des cellules dendritiques. Ces cellules seraient alors incubées in vitro avec le peptide pour que celui-ci se place sur leurs molécules de classe I. Elles pourraient également être incubées avec la protéine qu'elles captureraient, dégraderaient et présenteraient sur leurs molécules de classe I. On réinjecterait alors ces cellules au malade pour tenter de l'immuniser. Une troisième possibilité serait d'infecter les macrophages ou les cellules dendritiques avec des virus recombinants défectifs porteurs de la séquence codant le peptide antigénique. Des virus tels que les rétrovirus [30], les adénovirus [31] ou les virus apparentés à la vaccine [32] pourraient être utilisés. Des versions recombinantes des deux derniers virus pourraient également être injectées directement à des patients pour les immuniser.

Ces procédés visent une immunothérapie active, où la réponse contre l'antigène repose sur la participation in vivo du système immunitaire et s'accompagne d'une mémoire immunitaire capable d'une réponse plus rapide et plus forte lors d'injections ultérieures du même antigène. D'autres techniques visent à stimuler, in vitro en présence d'interleukine 2 , la prolifération de lymphocytes antitumoraux pour les injecter au malade en grande quantité. Ce procédé, appelé transfert adoptif, ne requiert pas la participation in vivo du système immunitaire et ne s'accompagne pas de mémoire immunitaire. Grâce à l'identification d'antigènes de rejet tumoral, la prolifération in vitro de ces lymphocytes peut se faire en présence de cellules présentatrices (lymphocytes B, cellules dendritiques ou macrophages) préalablement incubées avec le peptide antigénique ou inf ectées avec un virus recombinant de manière à présenter l'épitope tumoral. Ainsi chez un patient atteint de mélanome, des lymphocytes $\mathrm{T}$ infiltrant la tumeur ont été stimulés par des lymphocytes B incubés avec le peptide MAGE-1. Des CTL spécifiques dirigés contre le peptide MAGE-l présenté par HLA-Al ont été obtenus et amplifiés en vue d'un transfert adoptif [33]. Cette démarche devrait accroître la spécificité des lymphocytes injectés aux patients.

Il est probable que certains des modes d'immunisation que nous venons de décrire permettront d'obtenir des réponses de lymphocytes $T$ cytolytiques. En revanche, il paraît très difficile de prédire dans quelle mesure ces réponses seront capables d'éliminer complètement les cellules cancéreuses. En effet, ces cellules disposent de mécanismes qui leur permettront peut-être d'échapper aux réponses immunitaires provoquées par les immunisations [34]. Il semble cependant qu'un atout important sera la possibilité d'immuniser simultanément contre plusieurs antigènes portés par la même tumeur, car il sera plus difficile pour les cellules tumorales d'échapper à cette attaque multiple.

On ne peut donc pas affirmer avec certitude que l'immunisation contre des antigènes tumoraux définis va permettre de guérir des cancéreux. Mais l'avantage de la situation présente, c'est que pour la première fois nous allons pouvoir tenter une immunisation anticancéreuse sur une base bien définie. Celle-ci permettra de s'attaquer aux difficultés une par une en ayant chaque fois la possibilité d'analyser clairement les résultats

\section{Summary}

Tumor rejection antigens and specific immunotherapy of cancer

Experiments with mouse systems have shown that antigens recognized on tumors by cytolytic T lymphocytes can be the targets of immune responses that destroy the tumor cells without exerting harmful effects on normal tissues. In recent years, a number of genes that code for these antigens have been identified. The first gene studied, $M A G E-1$, coded for an antigen recognized by an autologous cytolytic T-cell clone. It belongs to a family comprising at least twelve members, but the function of the proteins encoded remains unknown. These genes have no expression in normal tissues (but testis and placenta); the tumoral peptides are presented to the immune system by specific HLA molecules. Some other tumoral antigens are not restricted to tumors but appear on normal melanocytes (tyrosinase, gp100...). Knowing these genes opens new possibilities in specific cancer immunotherapy.

\section{TIRÉS À PART}

T. Boon. 